\title{
Does mindfulness work?
}

\author{
Reasonably convincing evidence in depression and anxiety
}

\author{
Edo Shonin research director ${ }^{12}$, William Van Gordon principal investigator ${ }^{12}$, Mark D Griffiths \\ professor $^{1}$
}

${ }^{1}$ Psychology Division, Nottingham Trent University, Nottinghamshire NG1 4BU, UK, ; ${ }^{2}$ Awake to Wisdom Centre for Meditation and Mindfulness Research, Nottingham, UK

\begin{abstract}
Mindfulness has been defined as the process of paying attention to the present moment in a non-judgmental manner. ${ }^{1}$ In the early stages of mindfulness training, awareness of breathing is typically used as an attentional anchor to regulate ruminative thinking, ${ }^{2}$ but mindfulness encompasses much more than observing the breath. It derives from Buddhist practice and has been the subject of empirical investigation since the late 1970s, with over 700 scientific papers on mindfulness published in 2014. ${ }^{3}$
\end{abstract}

Evidence is most convincing for its use in the treatment of depression and anxiety. Meta-analyses assessing the efficacy of mindfulness in these two disorders have typically reported effect sizes in the moderate-strong to strong range (Cohen's d $\geq 0.5) .{ }^{4}$ However, some of the studies included in these meta-analyses have failed to control for a placebo effect, so it is unsurprising that meta-analyses with more stringent inclusion criteria report more modest outcomes. For example, a recent meta-analysis of 36 randomised controlled trials of mindfulness based stress reduction, mindfulness based cognitive therapy, and other mindfulness based interventions - each with an active control- reported small to moderate effect sizes $(\mathrm{d}=0.3-0.38)$ in the treatment of depression or anxiety after eight weeks of mindfulness training, with a reduction in effect size $(d=0.22-0.23)$ at three to six months' follow-up. ${ }^{6}$ Although these outcomes are more modest, they are comparable with results that would be expected from treatment with antidepressants in a primary care population, but without the associated toxicity. ${ }^{6}$ Consistent with these findings, the National Institute for Health and Care Excellence and the American Psychiatric Association advocate mindfulness based cognitive therapy for recurrent depression in adults. ${ }^{78}$ Some evidence suggests that mindfulness based interventions may have a role in treating other psychiatric conditions, including schizophrenia spectrum disorders, eating disorders, addiction disorders (both chemical and non-chemical), and sleep disorders. ${ }^{9}$ However, despite the fact that mindfulness has recently been included in the practice guidelines of the Royal Australian and New Zealand College of Psychiatrists as a non-firstline treatment for binge eating disorder in adults, ${ }^{10}$ there is arguably insufficient evidence from robustly designed randomised trials to support its use for conditions other than depression and anxiety.

Evidence is also accumulating that mindfulness may have a role in treating somatic conditions such as psoriasis, cancer, HIV infection, irritable bowel syndrome, heart disease, hypertension, lung disease, diabetes mellitus, and chronic pain. ${ }^{269}$ Evidence from randomised trials suggests that mindfulness based interventions (particularly mindfulness based stress reduction and cognitive therapy) are mildly to moderately efficacious in treating chronic pain $(d=0.33),{ }^{6}$ with possible applications for treating pain related disorders such as fibromyalgia. ${ }^{11}$ However, it is unclear whether mindfulness reduces the frequency and intensity of pain or simply improves patients' ability to cope with pain. ${ }^{11}$ With the exception of chronic pain and specific pain disorders, there is again insufficient high quality evidence to support mindfulness for treating somatic conditions.

\section{Unanswered questions}

As indicated above, various methodological problems limit the overall strength of the evidence on the efficacy of mindfulness. In particular, findings may be influenced by a form of "popularity effect." The growing popularity of mindfulness means that outcomes may be influenced by participants' belief that they are receiving a "fashionable" or proved psychotherapeutic technique. ${ }^{9}$ This is a difficult confounding variable to control for because it is almost impossible to blind patients from the fact they are using mindfulness techniques.

We also need greater clarity on whether positive outcomes are maintained over years, rather than just months, ${ }^{29}$ whether mindfulness interventions have any adverse effects, and the validity of the traditional view among contemplative traditions that sustainable improvements to health and wellbeing typically require daily mindfulness practice over many years (that is, they do not arise after attending just eight weekly classes of a few hours' duration). ${ }^{9}$

Furthermore, evidence is required to determine whether mindfulness in general or specific interventional approaches 
are most effective for a given illness (the Australian and New Zealand eating disorder treatment guidelines do not specify which mindfulness intervention is most suitable). Indeed, numerous interventions have been formulated with considerable variation in factors such as total participant-facilitator contact hours (including whether there is one-to-one contact), quantity and duration of guided mindfulness exercises, use of non-mindfulness psychotherapeutic techniques (such as psychoeducation or group discussion), inclusion of a full day silent retreat, emphasis on self practice (typically supported by a $\mathrm{CD}$ of guided mindfulness exercises), and use of other meditation techniques (such as yoga). ${ }^{2}$

Interventions also vary in how they define and operationalise mindfulness. For example, recent research has focused on second generation mindfulness interventions such as the eight week Meditation Awareness Training, which are based on the assumption that mindfulness is a psychospiritual rather than a purely psychological technique. ${ }^{12}$ Substantial variations in design and pedagogic approach make it difficult to generalise findings across the full spectrum of interventions.

Evidence is growing that mindfulness is effective in increasing perceptual distance from distressing psychological and somatic stimuli and that it leads to functional neuroplastic changes in the brain. ${ }^{13}$ However, the "fashionable" status of mindfulness among both the general public and the scientific community may have overshadowed the need to examine important methodological and operational issues concerning its efficacy.
Competing interests: We have read and understood BMJ policy on declaration of interests and declare that ES and WVG receive income from academic and trade publishers for writing books on mindfulness.

Provenance and peer review: Commissioned; externally peer reviewed.

1 Ludwig S, Kabat-Zinn, J. Mindfulness in medicine. JAMA 2008;300:1350-2.

2 Shonin E, Van Gordon W, Griffiths MD. Mindfulness-based interventions: towards mindful clinical integration. Front Psychol 2013:4:194.

3 American Mindfulness Research Association. AMRA database: mindfulness publications by year, 1980-2014. https://goamra.org/resources/.

4 Vollestad J, Nielson MB, Nielson GH. Mindfulness and acceptance-based interventions for anxiety disorders: a systematic review and meta-analysis. Br J Clin Psychol 2012;51:239-60.

5 Hofmann SG, Sawyer AT, Witt AA, et al. The effect of mindfulness-based therapy on anxiety and depression: a meta-analytic review. J Consult Clin Psychol 2010;78:169-83.

6 Goyal M, Singh S, Sibinga, EMS, et al. Meditation programs for psychological stress and well-being: a systematic review and meta-analysis. JAMA Intern Med 2014;174:357-68.

7 National Institute for Health and Care Excellence (NICE). Depression: management of depression in primary and secondary care. NICE, 2009.

8 American Psychiatric Association. Practice guideline for the treatment of patients with major depressive disorder. 3rd ed. APA, 2010

9 Van Gordon W, Shonin E, Griffiths MD. Mindfulness in mental health: a critical reflection. J Psychol Neuropsych Disord Brain Stim 2015;1:102.

10 Hay P, Chinn D, Forbes D, et al. Royal Australian and New Zealand College of Psychiatrists clinical practice guidelines for the treatment of eating disorders. Aust NZ J Psychiat 2014:48:1-62.

11 Lauche R, Cramer H, Dobos G, et al. A systematic review and meta-analysis of mindfulness-based stress reduction for the fibromyalgia syndrome. J Psychosom Res 2013;75:500-10.

12 Van Gordon W, Shonin E, Griffiths MD. Towards a second-generation of mindfulness-based interventions. Aust NZ J Psychiat 2015;49:591.

13 Taren AA, Gianaros PJ, Greco CM, et al. Mindfulness meditation training alters stress-related amygdala resting state functional connectivity: a randomized controlled trial. Soc Cogn Affect Neurosci 2015;1:1-11.

Cite this as: BMJ 2015;351:h6919

C BMJ Publishing Group Ltd 2015 